CALPHAD vo1.3, No.3, pp.171-173.

Pergamon Press Ltd. 1979. Printed in Great Britain.

\title{
CYBERNETIC PREDICTION OF SUPERCONDUCTING COMPOUNDS
}

\author{
E.M. Savitskii, V.B. Gribulya, N.N. Kiselyeva \\ A.A. Baikov Institute of Metallurgy \\ Moscow, Leninskii pr. 49
}

ABSTRACT. Common principles of the prediction of the existence of chemical compounds are reported. The data on the electronic structure of the atoms and other physical properties of chemical elements are used. The approach consists of utilization of certain ideas from cybernetics, from the field of learning types of computer systems to the prediction of physical-chemical characteristics of various materials The results of the prediction of the existence of binary and ternary phases, promising for synthesis of superconductors are cited. The attempts to estimate a critical temperature of superconductive transition of predicted compounds are discussed.

The phenomenon of superconductivity, discovered at the beginning of our century has been applied in technology more and more widely from year to year. Invention of materials, capable of retaining thesuperconducting properties at high magnetic fields and current densities, favours this application. So, the improvement of critical parameters of superconductors is a very important problem in the metallurgy of superconducting materials.

Physical theories of solids, while explaining the phenomenon of superconductivity itself cannot, at present, predict chemical composition of compounds with high critical temperature $\left(\mathrm{T}_{\mathrm{k}}\right)$, high critical magnetic field $\left(\mathrm{H}_{\mathrm{k} 2}\right)$, and high critical current ( $\left.\mathrm{I}_{\mathrm{k}}\right)$.

Prediction of new compounds, considered to be the most promising from the viewpoint of high $\mathrm{T}_{k}, \mathrm{H}_{\mathrm{k} 2}$, and $\mathrm{I}_{\mathrm{k}}$, cstimation of their critical temperatures - is the problem, which has been solved by the authors with the help of cybernetic methods of computer learning.

The basis of our approach is an automatic disclosure of empirical regularities from experimental data and further extrapolation and interpolation of these regularities to non-investigated systems. Computer learning is an analysis of information about an investigated physical-chemical system (i.e. about the existence of a phase with a certain crystalline structure and about temperatures of superconducting transition) performed in order to reveal regularities, and relating the possibility of forming the phase (having a certain crystalline lattice or certain $T_{k}$ range) with component properties of the physical-chemical system (1).

In this research the learning is considered as an active experiment. Thus a fixed part of information, formed during the process of computer learning is taken out in the form of a secondary description of chemical elements. Initial data on the elements constitute a primary description. The secondary description has the form of a matrix $M=a_{i j}$, where $a_{j j}$ is the matrix element or $j$-th value of the parameter for the $i-t h$ chemical element. Eacl, $j-t h$ column of the matrix is considered as a mathematical mapping of the chemical element number onto a new scale, related to its feature in the same way as $A$ or $B$ component of Al5 compound is related to stoichiometric formula $A_{m} B_{n}(m>n ;$ as a rule, m/n = 3/1). The type of crystalline lattice, A15 in particular, is assumed to be related to the properties of a given substances' class including the characteristics of superconductivity. So, we used this assumption for search of the relation between $T_{k}$ value and the parameters of the secondary description.

Thus the parameters of the secondary description are specific features of A15 phase components. A logically related set of these features determining the phase type, must describe in a proper way the properties determined by this type. In this case we considered mapping of $T_{k}$ onto the sum of parameters of the components $x=x_{A}+x_{B}$ secondary description for $A_{m} B_{n}$ compound of $A 15$ type. Here $x_{A}$ and $x_{B}$ are the elements of $M$ matrix, $a_{A j}$ and $a_{B j}$. 
The secondary description includes 8 parameters. We studied a two-dimensional mapping which is a section of multi-dimensional space by $T_{k}-X$ plane. The parameter $X$ was found to be the most interesting and had the following properties. All the known compounds of A15 type and those to be predicted are grouped into sets on $\mathrm{T}_{k}$ ordinates. The ordinates, in turn, form a characteristic set. Their external points are bounded by the envelope of the form close to an expotential function. Since the description components are not the results of direct measurements of certain physical properties but are logical representation of the initial description, their physical interpretation is complicated. In this case only coincidence or similarity of these components with the characteristics of known physical models may be ascertained.

The description component considered takes the following values $x_{j}$ for different chemical elements. For all p- and s- elements, which, as a rule, are B-components in Al5 tpe compounds the value of $x$ coincides with the group number in Mendeleev periodic table while for all $\mathrm{d}$ - and $f$-elements (except palladium) $x_{i}$ coincides with the number of external s-electrons, i.e. equals 1 or 2 . For palladium $x_{p d}=9$. Hence, the argument, $x$, when the $X$-dependence of $T_{k}$ distribution for alloys with A15 phase is considered,may take the values from 2 to 11. Compounds with maximum $T_{k}$ value are distributed on ordinates 4-6. The

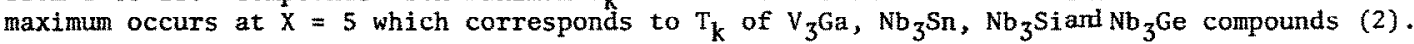

It is interesting to study superconductivity of A15 type phases not yet synthesized, but predicted, using the technology achievements. Only 45 compounds of this type out of 800 predicted ones get into the region of possible maximum. Among these there are 13 known compounds and 32 not yet synthesized ones on the basis of chromium, niobium, molybdenum, titanium, vanadium, hafnium, tantalum, tungsten. The second components of these compounds are boron, aluminum, gallium, indium, thallium, carbon, silicon, germanium, tin, lead. No double compound of Als type containing boron and carbon has been synthesized until now. It is probable, that these compounds under normal conditions will be metastable or not stable at all. However, in some cases instability may not prevent synthesis of alloys on the basis of unstable phases. They may be stabilized by doping additions and appropriate treatment.

On the basis of $\mathrm{T}_{k}$ value distribution in $\mathrm{X}$ coordinates one may assume that high $\mathrm{T}_{\mathrm{k}}$ values for double compounds with $\mathrm{A15}$ structure $1 \mathrm{i}$ e in the range $25-30^{\circ} \mathrm{K}$.

A promising group of compounds, having high $\mathrm{T}_{\mathrm{k}}$ and $\mathrm{H}_{\mathrm{k} 2}$ values, are Chevrel phases of $\mathrm{A}_{\mathrm{X}} \mathrm{Mo}_{6} \mathrm{~S}_{8}$ composition (A is an elerent of the Periodic system, $1 \leq \mathrm{x} \leq 3$ ) (3). These compounds are crystallized in the $\mathrm{Mo}_{3} \mathrm{Se}_{4}$ rhombohedral structure. More than 35 elements of the Periodic system may form Chevrel phases but only 7 of these compounds have $\mathrm{T}_{\mathrm{k}}$ higher than $4.2^{\circ} \mathrm{K}$.

We suggest that the possibility of Chevrel phase formulation with $T_{k}$ higher than 4.2 is mainly determined by physical-chemical properties of A and B elements. We choose two sets of properties: (1) electron structure and covalent radius of A and B elements; (2) magnetic susceptibility, first ionization potential, atomic radius and group number of $A$ and $B$ elements in the Periodic system. On the basis of experimental information the computer derived regularities of Chevrel phase formation with $\mathrm{T}_{\mathrm{K}}>4.2^{\circ} \mathrm{K}$ (depending on $A$ and $B$ elements' properties) areincluded in both the first and second set. Because of a strong correlation between element properties included in the second set, and the electron structure, i.e. the first set of the results of prediction with the use of the regularities obtained should, in general, coincide. In most cases the descrepancy is caused by the "noise" in a learning sampling, rough segmenting of continuous properties and low information capability of some initial paraneters.

According to our predictions, chromium, tungsten and rhenium used as $B$ component do not form Chevrel phase of $\mathrm{A}_{x} \mathrm{~B}_{6} \mathrm{~S}_{8}$ composition.

During the year after publication of our predictions (4) a compound $\mathrm{AgMo}_{6} \mathrm{~S}_{8}$ with $\mathrm{T}_{\mathrm{k}}=7.5^{\circ} \mathrm{K}(5)$ was obtained and studied and previous $1 \mathrm{y}$ unknown $\mathrm{U}_{1} 2_{2} \mathrm{Mo}_{6} \mathrm{~S}_{8}$ and $\mathrm{Th}_{1}, 2^{\mathrm{MO}} \mathrm{S}_{6} \mathrm{~S}_{8}$ Chevrel phases were synthesized. It allowed us to improve the cybernetic system further and to obtain corrected predictions. According to our data elements of II and III groups (Be, $\mathrm{Hg}, \mathrm{Ra}$ and $\mathrm{B}, \mathrm{Ac}$ and actinides) may also be used for obtaining $\mathrm{A}_{x} \mathrm{Mo}_{6} \mathrm{~S}_{8}$ phase with $\mathrm{Mo}_{3} \mathrm{Se}_{4}$ structure. According to our predictions Chevrel phases, in which $W$ and Re plays the role of A component, are very likely to exist. 
Thus, application of cybernetic prediction systems allows one to predict rather accurately the formation of new compounds, belonging to the most promising classes with the structure of $\mathrm{Al} 5$ and $\mathrm{Mo}_{3} \mathrm{Se}_{4}$ from the viewpoint of high superconducting characteristics. Empirical regularities, obtained with the help of computer training algorithm, allow one to estimate the temperature of superconducting transition for these phases. Computer predictions allow one to outline the ways of synthesizing new superconductors. Analysis of empirical dependences, simulated by the computer, enables one to suggest theoretical hypotheses about the reasons of formation of the compounds considered and about the nature of superconductivity in these phases.

\section{References}

1. Savitskii E.M., Gribulya V.B., Kiselyeva N.N., NBS SP-496, Applications of Phase Diagrams in Metallurgy and Ceramics, 1978, v. 2, 1139-1160.

2. Savitskii E.M., Gribulya V.B., Dok1. Acad. Nauk, USSR, 240, 316 (1978).

3. Fischer 0., App1. Phys., 16, 1 (1978).

4. Savitskii E.M., Kiselyeva N.N., Dok1. Acad. Nauk, USSR, 239, 405 (1978).

5. Flukiger R., Baillif R., Walker E., Mat. Res. Bull., 13, 743 (1978). 